\title{
REQUISITOS FUNCIONAIS PARA REGISTROS BIBLIOGRÁFICOS FRBR: UMA APRESENTAÇÃO
}

\author{
Fernanda Passini Moreno \\ Miguel Ángel Márdero Arellano
}

\begin{abstract}
Resumo
A área de estudos da Biblioteconomia, denominada representação descritiva, ou catalogação, tem um histórico de encontros de peritos para estabelecimento de padrões de descrição de documentos, no que tange às regras e formatos. Passados quarenta anos da Declaração dos Princípios de Paris, em 1961, teve início um re-exame de práticas e normas de catalogação, sob a responsabilidade de um grupo de estudos da Seção de Catalogação, Classificação e Indexação da IFLA-Internacional Federation Library Associations and Institutions - Federação Internacional de Associações e Instituições Bibliotecárias, materializado no relatório final: FRBR - Functional requirements for bibliographic records: final report, FRBR - Requisitos Funcionais para Registros Bibliográficos. Representando um avanço significativo na área de representação bibliográfica, publicado em 1998, apresenta conceitos e definições de entidades, relacionamentos e atributos, lançando um novo olhar sobre o objeto bibliográfico, centrado no usuário e suas ações. O modelo tornou-se objeto de teses, dissertações, grupos de estudos permanentes, tema de seminários e projetos de pesquisa, em nível internacional. Ao propor relacionamentos de diversas naturezas, os FRBR propõem o agrupamento de entidades com semelhanças, oferecendo um maior número de opções ao usuário que busca informações nos registros bibliográficos. Apesar de ser amplamente discutido, possuindo projetos de pesquisa para implementação do modelo em softwares bibliográficos, no exterior, no Brasil não existem registros de discussão a respeito. Este trabalho apresenta o modelo e algumas considerações como o início de um debate sobre o tema, que revela o futuro da descrição bibliográfica no cenário mundial e a criação do Código de Catalogação Internacional.
\end{abstract}

Palavras-chave

Registro bibliográfico; Descrição bibliográfica; Catalogação; FRBR; IFLA.

\section{FUNCTIONAL REQUIREMENTS FOR BIBLIOGRAPHIC RECORDS - FRBR: AN INTRODUCTION}

\begin{abstract}
Librarianship studies related with descriptive representation or cataloguing has an historic record of specialists meetings trying to establish standards of documents description, related to rules and formats. After 40 years of the Paris Declaration of Principles, in 1961, it started a re-examination of the cataloguing practices and rules, under the responsibility of a study group from the Cataloguing, Classification and Indexing Section of IFLA - International Federation Library Associations and Institutions. The result was the final report: FRBR - Functional requirements for bibliographic records. The FRBR represents a significant improvement in the bibliographic representation area. It was publish in 1982, presenting concepts and definitions of entities, relationships and attributes, giving a new look of the bibliographic object, focalizing users and their actions. The model became the object of theses, dissertations, permanent study groups, seminary issue and a research subject internationally. Proposing different kind of relationships, the FRBR proposed a gathering of similar entities, offering a larger number of options to the user who search for information into the bibliographic records. With all that international discussion and uses as a model for bibliographic software, in Brazil there is not a single record of FRBR been treated as an important issue. This paper presents the FRBR model and some considerations about it, as a proposition for discussion of the bibliographic description future worldwide and the creation of the International Cataloguing Code.
\end{abstract}

Key-words

Bibliographic record; Bibliographic description, Cataloguing; FRBR; IFLA.

(C) Revista Digital de Biblioteconomia e Ciência da Informação,Campinas, v .3, $n$ 1, p. 20-38, jul./dez. 2005-ISSN: 1678-765X. 


\section{INTRODUÇÃO}

A história da catalogação remonta às mais antigas bibliotecas que se tem conhecimento, com relatos da representação de documentos. Os catálogos manuais, como conhecemos hoje, só tiveram regras e estruturas definidas no século XIX, por Cutter $^{1}$ apesar de terem sido idealizados, inicialmente, por bibliógrafos e livreiros, interessados, apenas, na compilação de seus catálogos e bibliografias (BARBOSA, 1978). Os princípios estabelecidos por Cutter são fundamentais até hoje, como veremos adiante.

O presente artigo não pretende enfocar historicamente o catálogo, sua origem, etimologia, entre outros aspectos, posto que outros autores já o fizeram (BARBOSA, 1978; MEY, 1987, 1995, 1999, 2004; TAYLOR, 2004), nem é nosso objeto de estudo discutir normas e práticas. Restringimo-nos a comentar os encontros internacionais para estabelecimento de regras/normas de catalogação, que culminaram no desenvolvimento dos FRBR e em um futuro Código Internacional de Catalogação.

\section{ANTECEDENTES}

No século passado, a Declaração de Princípios da Catalogação, mais comumente conhecida por Princípios de Paris (1961), apresentou um resumo das regras desenvolvidas na Conferência Internacional sobre Princípios de Catalogação. Os Princípios marcaram época por se tratarem de um primeiro esforço de colaboração em nível internacional. Regem a catalogação até hoje e são usados, sempre que possível como filosofia para códigos nacionais.

Depois da primeira fase de automação dos catálogos, com a crescente troca de dados entre agências nacionais, se pôs em evidência a necessidade de um acordo internacional para a redação da descrição bibliográfica (BUIZZA, 2002). Padrões, normalizações e troca de

\footnotetext{
${ }^{1}$ Cutter, Charles A. Rules for a dictionary catalog. 4a. ed., rewritten Washington D.C.: Government Printing office, 1904.
}

(C) Revista Digital de Biblioteconomia e Ciência da Informação,Campinas, v .3, n 1, p. 20-38, jul./dez. 2005-ISSN: 1678-765X. 
dados/informações começam a ser desenvolvidos, como o formato MARC, desenvolvido nos EUA na década de 1960, e, no Brasil, a iniciativa CALCO, mais tarde chamada de rede Bibliodata $^{2}$.

Em 1968, surgem a AACR - Anglo-American Cataloging Rules (a AACR2 é publicada em 1978), representando o compromisso entre as novas idéias de catalogação e o que foi constatado como problemas reais em grandes bibliotecas que dispunham de catálogos extensos (BARBOSA, 1978).

O documento ISBD - International Standard Bibliographic Description (Descrição Bibliográfica Internacional Normalizada), publicado pela IFLA - Internacional Federation Library Associations and Institutions - Federação Internacional de Associações e Instituições Bibliotecárias, em 1971 tem seu início na Reunião Internacional de Especialistas em Catalogação, realizada em Copenhague (1969). Nesse documento estava sistematizada a ordem das informações bibliográficas, identificando elementos, dando ordem a eles e utilizando uma seqüência de pontuações padronizadas. É, até hoje, instrumento de comunicação internacional de informação bibliográfica. Outros documentos foram surgindo, inclusive para contemplar diferentes tipos de suportes, como a ISBD(G), para oito tipos de materiais.

Mey (1999, p. 7) aponta que o estabelecimento da ISBD partiu do estudo de práticas adotadas em oito bibliografias nacionais correntes, comparando-se tais práticas e buscando um elenco comum de elementos, porém não em virtude das necessidades dos usuários. Percebe-se também esse fato na descrição que Barbosa, op cit., nos dá sobre as AACR.

Parece-nos, portanto, que o foco da representação descritiva sempre se deu a partir das necessidades do usuário-meio, quais sejam, o bibliotecário e, não sob a perspectiva do usuário-fim. A finalidade de um registro bibliográfico, baseado no formato MARC, também apresenta incorreções - Hegna e Mutomaa (2002, p. 2) confirmam dizendo que:

\footnotetext{
${ }^{2}$ Em 1975, ficou decidido que o formato CALCO seria adotado em nível nacional para o processamento de dados bibliográficos referentes à produção científica brasileira. Para maiores detalhes, ver BARBOSA (1978).
} 
"central information is often recorded in a way more suitable for the human mind and eye, than a computer". A discussão e análise sobre os enlaces dos FRBR e MARC trata-se de tema já abordado por DELSEY (2002), e pelas autoras acima citadas.

A IFLA tem promovido padrões bibliográficos internacionais através da UBCIM Programme - Universal Bibliographic Control and International MARC, (Programa de Controle Bibliográfico Universal e MARC Internacional) e programas e atividades da Divisão para Controle Bibliográfico, que têm resultado em variados re-exames da teoria e prática da catalogação. Passados quarenta anos dos Princípios de Paris inicia-se uma re-avaliação na teoria e prática da catalogação, com apreciação internacional mais abrangente do que foi possível à época.

\section{FRBR}

A intensificação dos custos de catalogação; a contínua necessidade de economizar no processo de catalogação; o crescimento vertiginoso de publicações e a rápida proliferação de novos formatos e materiais com diversos novos métodos de acesso. Esses pontos, tão conhecidos dos bibliotecários, motivaram os participantes do seminário de Estocolmo, Suécia, em 1990, patrocinado pela IFLA UBCIM Programme e Divisão de Controle Bibliográfico. Dentre as várias recomendações do seminário, duas são a base para um reexame dos registros bibliográficos: a necessidade de se estabelecer um nível básico de funcionalidade para os registros bibliográficos em relação à variedade de usuários e de mídias e que as agências bibliográficas nacionais ficassem responsáveis por garantir que suas publicações saíssem em diversas mídias, fazendo, em conjunto, um estudo de necessidade dos usuários.

Ao longo de oito anos, o grupo de estudos oriundo da Seção de Catalogação e da Seção de Classificação e Indexação da IFLA, com a colaboração de consultores e de voluntários de várias nacionalidades, desenvolveu os FRBR, apresentando um relatório final em 1998, configurando uma recomendação para reestruturar os registros bibliográficos de maneira a refletir a estrutura conceitual de buscas de informação, levando em conta a diversidade

(C) Revista Digital de Biblioteconomia e Ciência da Informação,Campinas, v .3, $n$ 1, p. 20-38, jul./dez. 2005-ISSN: 1678-765X. 
de:

- usuários - usuários da biblioteca, pesquisadores, bibliotecários da seção de aquisição, publicadores, editores, vendedores;

- materiais - textuais, musicais, cartográficos, audiovisuais, gráficos e tridimensionais;

- suporte físico - papel, filme, fita magnética, meios óticos de armazenagem, etc. e,

- formatos - livros, folhas, discos, cassetes, cartuchos, etc. que o registro possa conter.

Os FRBR oferecem-nos uma nova perspectiva da estrutura e nos relacionamentos dos registros bibliográficos. A proposta dos FRBR é:

primeiro, fornecer um quadro estruturado, claramente definido, para relacionar dados registrados em registros bibliográficos às necessidades dos usuários destes registros. O segundo objetivo é recomendar um nível básico de funcionalidade para registros criados por entidades bibliográficas nacionais. (IFLA, 1998, p. 7).

No primeiro objetivo, percebe-se a intenção da proposta inovadora dos FRBR: que catálogos em linha, baseados no modelo, possam mostrar as relações bibliográficas mais claramente, de maneira útil ao usuário, de maneira que ele possa navegar em "espaços" de informações complexos, através das relações, de maneira que as informações nos registros, recuperadas através da expressão de busca do usuário, reflitam um apropriado "rol" de registros (BEACOM, 2003).

Para responder às necessidades dos usuários, os Requisitos Funcionais para Registros Bibliográficos são definidos em relação às seguintes tarefas genéricas realizadas pelos usuários quando fazem buscas em bibliografias nacionais e catálogos de bibliotecas, ou os 
utilizam, chamadas user tasks:

- uso dos dados para encontrar materiais que correspondam aos critérios estabelecidos para a busca do usuário;

- uso dos dados recuperados para identificar uma entidade;

- uso dos dados para selecionar uma entidade adequada às necessidades do usuário;

- uso dos dados para encomendar, adquirir, ou obter acesso à entidade descrita (IFLA, 1998, p. 8).

Nota-se que as tarefas genéricas acima descritas são fortemente inspiradas nos três objetivos do catálogo, propostos por Cutter (MEY, 1987; RILEY, 2004; TAYLOR, 2004), quais sejam:

1) Permitir a uma pessoa encontrar um livro do qual ou: a) o autor; b) o título; c) o assunto seja conhecido;

2) Mostrar o que a biblioteca possui: d) de um autor determinado; e) um assunto determinado; f) em um tipo dado da literatura;

3) Para ajudar na escolha de um livro: g) de acordo com sua edição (bibliograficamente); h) de acordo com seu caráter (literário ou tópico).

No segundo objetivo, é proposto um nível básico de funcionalidade. Este nível mínimo ou básico que o Relatório recomenda se pautou em análises de entidades relatadas como necessárias para os diversos tipos de usuários que contribuíram com o grupo de estudos durante os oito anos que esteve em debate.

Além disso, a descrição dos elementos básicos do modelo desenvolvido para o estudo entidades, atributos e relações - se derivaram de uma análise lógica dos dados que se acham tipicamente refletidos nos registros bibliográficos. As principais fontes utilizadas na análise incluem:

- as ISBDs (Descrições Bibliográficas Internacionais Normalizadas),

- as Guidelines for Authority and Reference Entries (GARE) (Diretrizes para Entradas de Cabeçalhos Autorizados e Remissivas), 
- as Guidelines for Subject Authority and Reference Entries (GSARE) (Diretrizes para Entradas de Cabeçalhos Autorizados de Assunto e Remissivas) e

- o UNIMARC Manual.

Dados adicionais foram coletados de outras fontes, como as AITF Categories for the Description of Works of Art (Categorias AITF para a Descrição de Obras de Arte) (IFLA, 1998, p. 4).

A técnica de análise de entidades, atributos e relações, qual seja, o Modelo EntidadeRelacionamento, tem sua autoria na década de 70 (CHEN, 1976) e posteriormente desenvolvido e ampliado.

Modelos de dados são um conjunto de conceitos utilizados para descrever um banco de dados. O modelo E-R é um modelo lógico com base em objetos, e a identificação de entidades e relacionamentos é entendida como a captura da semântica dos dados, para projetar um banco de dados. Este modelo não visa à implementação e sim à modelagem/representação dos dados. A partir desta modelagem, é possível implementar um banco de dados em outros modelos de dados: orientado a objeto, relacional, etc.

Entidade é aqui entendida como uma "coisa" ou um "objeto" no mundo real que pode ser identificada de forma unívoca em relação a todos os outros objetos. Uma entidade pode ser concreta ou abstrata. Por sua vez, atributos são as diversas características que um tipo de entidade possui, ou propriedades descritivas de cada membro de um conjunto de entidades. Um relacionamento "é uma associação entre uma ou várias entidades” (CHEN, 1990, p. 21-24).

Tendo a intenção de servir como modelo de referência, os FRBR operam no nível conceitual, não levando a análise dos requisitos ao nível necessário para um modelo de dados desenvolvidos de forma completa. Os FRBR não são exatamente um modelo de 
dados, por serem “[...] demasiado abstratos e genéricos para serem um modelo de dados: nenhuma base de dados prática poderia realmente ser desenvolvida exclusivamente baseados naquelas entidades, atributos, e relacionamentos que são definidos nos FRBR”(IFLA, 2003) ${ }^{3}$.

Esses elementos são agora apresentados:

\section{Entidades}

Ao todo, são listadas dez entidades, dividas em três grupos:

As primeiras quatro entidades pertencem ao primeiro grupo, que compreende as entidades que são produto de trabalho intelectual ou artístico: obra, expressão, manifestação e item.

Obra é uma entidade abstrata, uma criação intelectual ou artística distinta. A entidade Expressão de uma obra é a realização intelectual ou artística especifica que assume uma obra ao ser realizada, excluindo-se aí aspectos da alteração da forma física.

Uma Manifestação é a materialização de uma expressão de uma obra, ou seja, seu suporte físico, que podem ser livros, periódicos, kits multimídia, filmes, etc, que é representada pelo Item, um único exemplar de uma manifestação. As duas últimas entidades refletem a forma física, são entidades concretas, enquanto as duas primeiras refletem o conteúdo intelectual ou artístico.

O segundo grupo apresenta mais duas entidades, aqueles responsáveis pelo conteúdo intelectual ou artístico, pela produção física e disseminação, ou pela guarda das entidades do primeiro grupo: pessoa ou entidade coletiva (ibidem).

\footnotetext{
${ }^{3}$ A partir do site: $\mathrm{http}: / /$ www.ifla.org/VII/s13/wgfrbr/faq.htm.
} 
As quatro últimas entidades são elencadas no terceiro grupo, um conjunto adicional de entidades que servem como assuntos de obras: conceito, objeto, evento e lugar.

Alguns exemplos concretos de entidades, para maior compreensão: os primeiros, a partir de Tillett (2003, p.10), o quadro da figura 1, adaptado de Beacom (2003).

Quando nos referimos a "um livro", estamos descrevendo um objeto físico, com folhas de papel e demais características que lhe são próprias. Para os FRBR, isso é um item. Também quando nos referimos a um livro, podemos querer dizer uma "publicação", conhecendo seu ISBN, inclusive. Para os FRBR, trata-se de uma manifestação. No entanto, se dissermos, "um livro, que é tradução de...", tendo em mente um texto em particular, em uma língua específica, estamos diante de uma expressão.

Nota-se, na figura 1, duas obras distintas: um romance literário e um filme.Um romance, enquanto obra, pode ser "realizado" de várias maneiras: o texto original, o texto traduzido para outra(s) língua(s), uma edição ilustrada. São várias expressões de uma obra original. Qualquer mudança no conteúdo intelectual ou artístico constitui mudança na expressão. Assim, se um texto é revisto ou modificado, a expressão daí resultante é considerada como uma nova expressão, não importando quão aparentemente pequena possa ser a modificação. Nesse exemplo, temos a expressão "tradução" da obra, que "está contida” em papel.

A expressão "edição ilustrada" "está contida" nas manifestações em papel, no formato Adobe Reader (pdf), uma página da internet.

Um exemplo de item é um livro autografado, mas poderia ser uma página da internet impressa ou o documento em formato Adobe arquivado em um computador. O item é físico, mesmo que virtual. O item é a exemplificação da manifestação.

(C) Revista Digital de Biblioteconomia e Ciência da Informação,Campinas, v .3, $n$ 1, p. 20-38, jul./dez. 2005-ISSN: 1678-765X. 


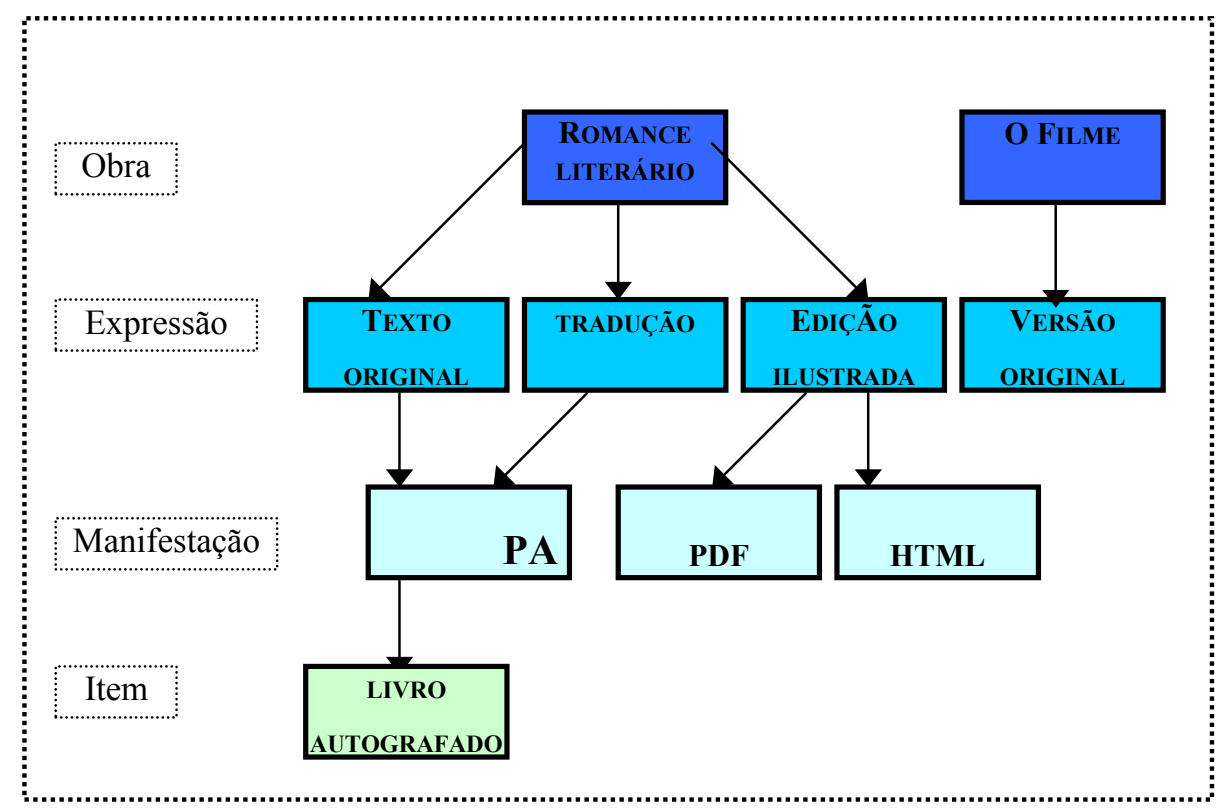

FIGURA 1: Exemplos de entidades do grupo1

Fonte: Adaptado de Beacom (2003).

Um filme, "o filme", é uma obra, entidade abstrata - só se realiza através da expressão: versão original. Versões dubladas ou legendadas de um filme são considerados apenas como diferentes expressões da mesma obra original. A expressão filme "está contida em" manifestações, que poderiam estar no exemplo: em DVD ou em VHS.

Quando a modificação da obra envolve um grau significativo de esforço intelectual ou artístico, o resultado é visto, nos FRBR, como uma nova obra. Portanto, hipoteticamente, se o romance literário do exemplo acima teve adaptação para o cinema e tornou-se uma obra cinematográfica, este filme é uma nova obra. 


\section{RELACIONAMENTOS}

No contexto do modelo, relacionamentos servem com um veículo para descrever ligações entre uma entidade e outra, e conseqüentemente como um meio de ajuda ao usuário para "navegar" no universo que é representado na bibliografia, catálogo, ou [em um] banco de dados bibliográfico (IFLA, p. 56).

Há relacionamentos bibliográficos implícitos, chamados nos FRBR relacionamentos de nível alto, ou de primeiro nível: uma obra é realizada através da expressão, e pode sê-lo em mais de uma, como no exemplo do romance literário. No entanto, uma expressão é a realização de uma e apenas uma obra.

Uma expressão pode ser materializada em uma ou mais de uma manifestação; da mesma forma uma manifestação pode materializar uma ou mais de uma expressão. Uma manifestação, por sua vez, pode ser exemplificada por um ou mais de um item; porém um item pode exemplificar uma e apenas uma manifestação. A figura 2 esquematiza essas relações.

A relação de responsabilidade associa as entidades acima descritas (primeiro grupo) às entidades do segundo grupo: pessoa e entidade coletiva. Estas podem criar uma obra, realizar uma expressão, produzir uma manifestação e possuir um item.

As relações de assunto exemplificam as relações existentes entre uma obra e as entidades do primeiro e segundo grupos. Uma obra pode ter como assunto um(a) ou mais obra, expressão, manifestação, item, pessoa e, ou, entidade coletiva.

Definir obra como entidade também permite estabelecer relações indiretas entre expressões da mesma obra, nos casos em que se está incapacitado de desenhar relações diretas entre expressões individuais; e assim sucessivamente com as demais entidades. Relacionar expressões de uma obra indiretamente, ao relacionar cada expressão à obra que realiza, é

(C) Revista Digital de Biblioteconomia e Ciência da Informação,Campinas, v .3, n 1, p. 20-38, jul./dez. 2005-ISSN: 1678-765X. 
com freqüência o meio mais eficiente de agrupar expressões relacionadas (IFLA, p.18).

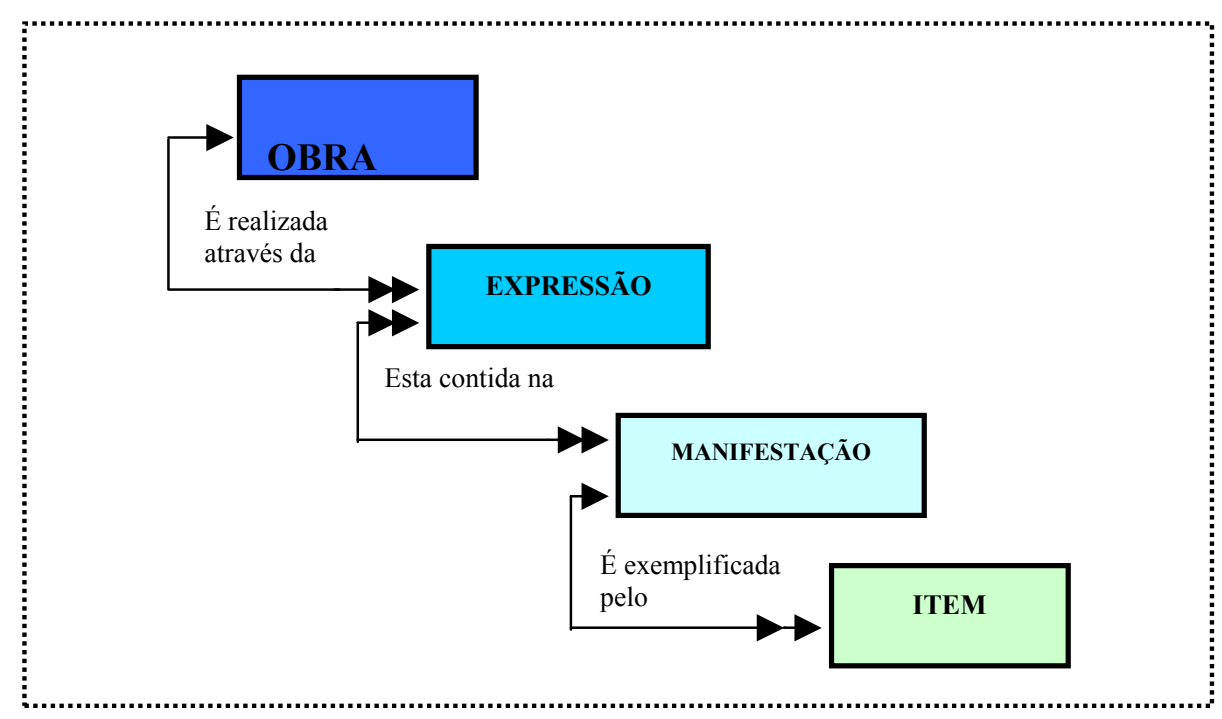

FIGURA 2: Entidades do Grupo 1 e Relações Bibliográficas Primárias Fonte: FRBR, traduzido por MEY (1999)

Outras relações estão presentes no modelo.

Nos FRBR a importância dos relacionamentos é o foco nas tarefas do usuário. Os relacionamentos todo/parte e parte-para-parte também estão nos FRBR. Descrevê-los, todos seria inviável no presente artigo, pela sua complexidade e inclusive por haver questões interessantes quanto à sua origem e desdobramentos, que sugerem a realização de um trabalho enfocando apenas esse assunto. 
Sumarizando os relacionamentos, temos: relacionamentos entre obra, expressão, manifestação e item, relacionamentos entre pessoas e entidades coletivas, relacionamentos de assunto, acima apresentados.

Temos ainda, relacionamentos associados às quatro primeiras entidades (obra, expressão, manifestação e item) que operam mais especificamente entre instâncias designadas de entidades, quais sejam:

Quadro 1 - Relacionamentos presentes nos FRBR

\begin{tabular}{|c|c|}
\hline Relacionamento de Obra-para-Obra & Relacionamentos de Todo/Parte no nível da Obra \\
\hline Relacionamentos Expressão-para-Expressão & $\begin{array}{c}\text { Relacionamentos Todo/Parte no nível da } \\
\text { expressão }\end{array}$ \\
\hline $\begin{array}{c}\text { Relacionamentos Manifestação-para- } \\
\text { Manifestação }\end{array}$ & $\begin{array}{c}\text { Relacionamentos de Todo/Parte no nível da } \\
\text { manifestação }\end{array}$ \\
\hline Relacionamentos de Item-para-Item & Relacionamentos de Todo/Parte no nível do item \\
\hline Relacionamentos Expressão-para-Obra & Relacionamentos Manifestação-para-Item \\
\hline
\end{tabular}

Fonte: FRBR. Tradução própria

Um exemplo concreto seria o tipo de relacionamento classificado como "reprodução". Entre duas manifestações, é traçado o relacionamento "tem uma reprodução" e "é uma reprodução de". O quadro abaixo, retirado dos FRBR esquematiza essa relação. 


\section{Quadro 2 - Relacionamento manifestação-para-manifestação}

\begin{tabular}{|l|l|}
\hline \multicolumn{1}{|c|}{ Tipo de relacionamento } & \multicolumn{1}{|c|}{ Manifestação } \\
\hline Reprodução & $\begin{array}{l}\text { Reprodução } \\
\text { Microrreprodução } \\
\text { Reimpressão } \\
\text { Tem uma reprodução -> } \\
<- \text { é uma reprodução de }\end{array}$ \\
& $\begin{array}{l}\text { Reimpressão em photo-offset } \\
\text { Facsimile }\end{array}$ \\
\hline
\end{tabular}

Fonte: FRBR. Tradução própria

\section{Atributos}

Às entidades, são associados atributos, características, similares a elementos de dados, que podem ser diretamente ligados à entidade ou externos à entidade. Os atributos inerentes às entidades se referem às características físicas, aspecto formal que caracterizam uma manifestação, ou outros identificados através do exame do item (por exemplo, informações na capa, na página de rosto, colofão, etc). Os atributos externos, ou imputados a uma entidade, compreendem os identificadores daquela entidade e informações contextuais, como por exemplo, o número no catálogo temático, ou o contexto em que a obra foi realizada. Geralmente, requerem o uso de outras fontes para estabelecê-los (GUERRINI, 2001; BEACOM, 2004).

Atributos ou metadados são como os elementos de descrição bibliográfica propriamente ditos. Nos FRBR, são categorizados de acordo com as entidades, incluindo os mais diferentes tipos de materiais e suas características. Abrange desde registros sonoros tendo como atributos modalidade de captação, meio físico, extensão do suporte, velocidade de execução (no caso de uma manifestação), até objetos cartográficos, por exemplo, que possuem, na expressão, como atributos: escala, projeção, técnica de apresentação, entre outros. Um recurso eletrônico de acesso remoto, por exemplo, apresenta como atributos as 
características do arquivo, forma de acesso, endereço de acesso, e assim por diante.

O usuário formula suas buscas através dos atributos: autor de determinada obra, título desta, editora e assim sucessivamente.

A principal contribuição em definir as entidades é poder estabelecer as distinções entre uma obra e outra, entre uma obra e sua expressão, entre duas expressões, através de atributos, e usar as diferenças nestes atributos para demonstrar as diferenças no conteúdo intelectual ou artístico (IFLA, 1998, p.18).

\section{COMENTÁRIOS FINAIS}

Após a apresentação dos FRBR, temos que: as tarefas de usuário (user tasks) apresentam conceitos expandidos da filosofia que regem a confecção de catálogos. As entidades, como descritas, refletem uma nova percepção sobre o objeto bibliográfico: ao distinguir o conteúdo da forma física, e relacioná-los, agrupam-se entidades com semelhanças, porém, de forma mais abrangente do que as opções de um catálogo comum (mesmo que eletrônico) podem oferecer. Ao propor relacionamentos de diversas naturezas, novamente são agrupadas entidades com semelhanças, oferecendo um maior número de opções ao usuário, como vemos "os relacionamentos refletidos no registro bibliográfico proporcionam [uma] informação adicional que ajudam o usuário a fazer novas conexões entre a entidade encontrada e outras entidades que se relacionam com aquela entidade" (IFLA, p. 56).

Ao detalhar os atributos das entidades, os FRBR propõem uma descrição bibliográfica muito completa e abrangente, usando os dados adicionais, mais uma vez, para relacionar objetos que guardem alguma semelhança.

À época da publicação, parte do texto, presente nos FRBR, julgava o estudo ainda possível de expansão, não se pretendendo completo, e que poderia ser a base para uma revisão de formatos, como o MARC.

(C) Revista Digital de Biblioteconomia e Ciência da Informação,Campinas, v .3, n 1, p. 20-38, jul./dez. 2005-ISSN: 1678-765X. 
A IFLA continua monitorando a aplicação e promovendo seu uso e desenvolvimento, mantendo através da Seção de Catalogação um grupo permanente de estudos, para expansão do modelo, aplicação na prática da catalogação, ensino e treinamento, em outras atividades.

Como os FRBR se tornaram objetos de pesquisa envolvendo as mais diversas abordagens, resultando em teses, dissertações, seminários de alcance internacional, entre outros, possui uma bibliografia constantemente atualizada de textos completos, artigos, livros, em linha ou impressos. A bibliografia ${ }^{4}$ abrange as traduções para sete línguas, aspectos teóricos, (algumas questões, mesmo após aprovação do relatório final, ainda estão em aberto); os impactos nos padrões vigentes de catalogação; aspectos relativos à implementação, na forma de softwares específicos, entre tantos outros assuntos a eles relacionados. É mantida, ainda, uma ativa lista de discussões ${ }^{5}$ moderada por Patrick LeBœuf.

Os FRBR estão acima de códigos e normas, porém baseadas nelas, como as ISBDs, além de servirem como base para reflexão sobre a estrutura do Código de Catalogação AngloAmericano - AACR2 e das ISBDs.

O vocabulário preciso pode auxiliar designers/fabricantes de sistemas irem ao encontro das necessidades dos usuários. Atualmente (2005), há uma ampla série de estudos a respeito da aplicação, implementação dos FRBR, envolvendo instituições como OCLC (On Line Computer Library Center), com quatro projetos em andamento; VTLS (Virginia Tech Library System); Indiana University (Digital Music Library Project), entre outros projetos.

Um software de gerenciamento de registros bibliográficos (ou software bibliográfico), ancorado no modelo, ofereceria aos usuários do catálogo mais opções do que um software

\footnotetext{
${ }^{4}$ Disponível em: http://www.ifla.org/VII/s13/wgfrbr/bibliography.htm .

${ }^{5}$ Disponível em: http://www.ifla.org/VII/s13/wgfrbr/listserv.htm. Inscrição a partir de: http://infoserv.inist.fr/wwsympa.fcgi/info/frbr.
} 
comum: o exemplo de desenvolvimento deste, pela $\operatorname{VTLS}^{6}$, mostra quão amplas ficam as possibilidades de escolha do usuário, a partir dos relacionamentos.

Concluímos, afirmando que o lançamento de diretrizes para um Código Internacional de Catalogação, ICC, no encontro de peritos patrocinado pela IFLA, 2003 (IFLA Meeting of Experts on an International Cataloguing Code - IME ICC), e seu documento base ${ }^{7}$, lançado no segundo encontro em 2004, já incorporando os conceitos dos FRBR, exemplificam o impacto do modelo e configuram o novo cenário da catalogação mundial.

Sugerimos que se inicie um debate nacional a respeito, e que os usos, a prática e o ensino de catalogação se pautem pelos mais avançados conceitos e aplicações em desenvolvimento na área.

\section{REFERÊNCIAS}

BARBOSA, A. P. Novos rumos da catalogação. Rio de Janeiro: BNG/Brasilart, 1978.

BEACOM, Matthew. The once \& future catalog: the FRBR model, users and catalogs. Disponível em:

$<$ http://www.library.yale.edu/ mbeacom/talk/Once\%20and\%20Future $\% 20$ Catalog2.ppt $>$ Acesso em: 02 nov. 2004.

BUIZZA, Pino. Dai Principi di Parigi a FRBR. "Bibliotime", v. 5, n. 1, 2002. Disponível em: <http://www.spbo.unibo.it/bibliotime/num-v-1/buizza.htm $>$. Acesso em 13 out. 2004.

CHEN, P. Modelagem de dados: a abordagem entidade-relacionamento para projeto lógico. Tradução de Cecília Camargo Bartalotti. São Paulo: Mcgraw Hill, 1990.

DELSEY, Tom. Functional analysis of the MARC 21 bibliographic and holdings

formats. Washington:Library of Congress, January 4, 2002 [cited 6 February 2002].

Disponível em:

$<\underline{\text { http://www.loc.gov/marc/marc-functional-analysis/functional-analysis.html }}>$ Acesso em: 10 fev. 2005.

\footnotetext{
${ }_{7}^{6}$ Disponível em: http://www.vtls.com/documents/FRBR.PPT.

${ }^{7}$ Disponível em: http://www.loc.gov/loc/ifla/imeicc/papers-sp.html.
} 
FEDERAÇÃO INTERNACIONAL DE ASSOCIAÇÕES E INSTITUIÇÕES BIBLIOTECÁRIAS. Grupo de estudos sobre Requisitos Funcionais para os Registros Bibliográficos. FRBR: relatório final. Tradução de Eliane Serrão Alves Mey. Documento não publicado.

FINAL Glossary IME ICC. FRBR Glossary [2004] .Disponível em:

<http://www.ddb.de/news/pdf/glossary_april_2004.pdf> . Acesso em: 02 nov. 2004.

GUERRINI, Mauro. FRBR in sintesi. Napoli: Università degli Studi di Napoli Federico II, Dipartimento di Discipline Storiche "Ettore Lepore", [2001?]. Disponível em:

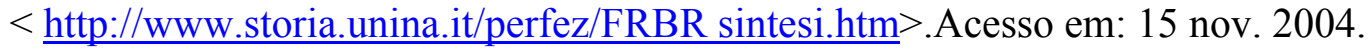

HEGNA, Knut; MUTOMAA, Eeva. Data minig MARC to find: FRBR? IFLA Cataloguing Section. Glasgow, 2002. Disponível em: http://folk.uio.no/knuthe/dok/frbr/datamining.pdf.

IFLA Cataloguing Section - FRBR Review Group. FRBR Bibliography.

Disponível em: < http://www.ifla.org/VII/s13/wgfrbr/bibliography.pdf $>$. Acesso em: 27 abr. 2004.

IFLA Study Group on the Functional Requirements for Bibliographic Records. Functional requirements for bibliographic records: final report. München : K. G. Saur, 1998

IFLA Cataloguing Section - FRBR Review Group. Frequently Asked Questions about FRBR. [version 9.0 latest, revision, oct. 2004]. Disponível em:

$<$ http://www.ifla.org/VII/s13/wgfrbr/faq.htm>. Acesso em: 02 nov. 2004.

MEY, Eliane S. A. Acesso aos registros sonoros: elementos necessários à representação bibliográfica de discos e fitas. São Paulo, 1999. Tese (Doutorado) - Universidade de São Paulo.

\section{. Bibliotheca Alexandrina. Campinas, Revista Digital de}

Biblioteconomia e Ciência da Informação,v.1, n. 2, p. 71-91, 2004.

. Catalogação e descrição bibliográfica: contribuições a uma teoria. Brasília: Associação dos Bibliotecários do Distrito Federal, 1987. 201p. Originalmente apresentada como dissertação de Mestrado, Universidade de Brasília, 1986.

. Introdução à catalogação. Brasília: Briquet de Lemos, 1995.

RILEY, Jenn. FRBR: or, How I learned to stop worrying and love the model. [jan 2004] Disponível em:

$<\underline{\mathrm{http}}$ //www.dlib.indiana.edu/ jenlrile/presentations/bbfall04/frbr/frbrBB.ppt $>$. Acesso em: 2 nov. 2004. 
TAYLOR, Arlene G. Wynar's introduction to cataloging and classification. 9. ed. London: Libraries Unlimited, 2004.

TILLETT, Barbara. FRBR: Functional requirements for bibliographic records.

Technicalities, v. 23, n. 5, set./out. 2003, p. 10-13.

Miguel Ángel Márdero Arellano

Doutorando em Ciência da Informação, CID, UnB

e-mail: miquel@ibict

Fernanda Passini Moreno

Graduada em Biblioteconomia e Ciência da Informação, UFSCar. Mestranda em Ciência da Informação, CID,UNB. Bolsista CAPES.

e-mail: fernandam@unb.br

Artigo aceito para publicação em: 07/ 2005 